\title{
Sum List Coloring $2 \times n$ Arrays
}

\author{
Garth Isaak* \\ Department of Mathematics \\ Lehigh University, Bethlehem, PA 18015 \\ gisaak@lehigh.edu
}

Submitted: September 30, 2001; Accepted: May 7, 2002.

MR Subject Classifications: 05C15

\begin{abstract}
A graph is $f$-choosable if for every collection of lists with list sizes specified by $f$ there is a proper coloring using colors from the lists. The sum choice number is the minimum over all choosable functions $f$ of the sum of the sizes in $f$. We show that the sum choice number of a $2 \times n$ array (equivalent to list edge coloring $K_{2, n}$ and to list vertex coloring the cartesian product $\left.K_{2} \square K_{n}\right)$ is $n^{2}+\lceil 5 n / 3\rceil$.
\end{abstract}

List coloring has been well-studied in recent years (see for example surveys in [1], [7], $[9],[11]$ ). The vertices of a graph (often a line graph) are given color lists and we seek to determine if there is a proper coloring using colors from the lists. Typically one seeks the minimum value $k$ such that for every choice of lists of size $k$ there is a proper coloring. In this case we call the graph $k$-choosable. More generally the list size for each vertex can be specified and one can determine if for every choice of lists of the specified sizes there is a proper coloring. The general problem here would be to characterize which list size assignments allow a proper coloring for every choice of lists of the given sizes. For the case of line graphs of complete bipartite graphs this is called the generalized Dinitz problem in [5] and it is noted that it 'was proposed independently by (at least) H. Taylor and D. Knuth (personal communications) and the author.' (The 'author' referred to in the previous sentence is Galvin.) The general characterization problem for line graphs of trees (and more generally for vertex coloring graphs with every block a clique) is solved in another paper [8] by the author of this paper.

For the $k$-choosability problem we seek to minimize the maximum list size. Here we will examine another special case of the generalized problem where we seek to minimize the average list size (by minimizing the sum of the list sizes). While there have been a number of generalizations and variations on choosability as well as results with sufficient conditions for choosability based on degrees, it seems that the problem of minimizing the sum of the list sizes has not been examined.

\footnotetext{
*Partially supported by a grant from the Reidler Foundation
} 
As noted in the abstract, properly coloring the edges of the complete bipartite graph $K_{2, n}$ corresponds to properly coloring the vertices of the cartesian product $K_{2} \square K_{n}$ and also corresponds to properly coloring the cells of a $2 \times n$ array with distinct colors in each row and each column. We will adopt the last perspective in this paper.

For a collection $\mathcal{C}=\{C(i, j) \mid i=1,2, \ldots, m$ and $j=1,2, \ldots, n\}$ of lists we will say that the $m \times n$ array is $\mathcal{C}$-colorable if we can choose colors $c_{i, j} \in C(i, j)$ for the cells $(i, j)$ of the array such that the colors selected for each row and column are distinct (that is, $c_{i, j}=c_{r, s} \Rightarrow i \neq r$ and $j \neq s$ ). We will call such a choice a proper $\mathcal{C}$-coloring. For $1 \times n$ arrays we will write $C(i)$ rather than $C(1, i)$. Consider a size function $f$ : $\{1,2, \ldots, m\} \times\{1,2, \ldots, n\} \rightarrow \mathbf{Z}$. We will say that the $m \times n$ array is $f$-choosable if for every $\mathcal{C}$ with $|C(i, j)|=f(i, j)$, the array is $\mathcal{C}$-colorable. For convenience we allow $f(i, j) \leq 0$. If any $f(i, j) \leq 0$ then we will say that the array is not $f$-choosable. We will call $\sum_{i=1}^{m} \sum_{j=1}^{n} f(i, j)$ the size of $f$. Then, the sum choice number of the $m \times n$ array is the minimum size of a choosable $f$.

Theorem 1 The sum choice number of the $2 \times n$ array is $n^{2}+\lceil 5 n / 3\rceil$.

Proof: This will follow immediately from Lemmas 2 and 3 in Sections 2 and 3. $\square$.

We will use an array notation to represent size functions $f$. So for example, $\begin{array}{rr}2 & 3 \\ 1 & 2\end{array}$ represents the function $f(1,1)=f(2,2)=2, f(1,2)=3$ and $f(2,1)=1$.

We will say that a collection of lists is initial if every list is of the form $\{1,2, \ldots, s\}$. List coloring graphs with the restriction that every list is initial is essentially graph coloring. For sum list coloring, if the lists are restricted to be initial, then we get sum coloring (see, for example, [2] for more on this problem). Finding the minimum sum for a coloring is NP-complete even for line graphs of bipartite graphs with maximum degree three [6].

We will refer to greedy coloring with respect to a given ordering of the cells as the coloring obtained by sequentially coloring the cells in the given order, choosing the smallest color at each step that will not violate the conditions for a proper list coloring. Greedy coloring will produce a proper list coloring if, when each cell is colored, the size of its list is greater than the number of previously colored cells in the same row or column having lists that have non-empty intersection with the cell being colored.

\section{Lower Bound}

We first show that for the $1 \times n$ array the sum choice number is $1+2+\cdots+n=n(n+1) / 2$. If the list sizes are $1,2, \ldots, n$, then greedy coloring with the ordering determined by smallest to largest list size works. Consider any $f$ that is choosable for the $1 \times n$ array. We may assume that the sizes are arranged so that $f(1) \leq f(2) \leq \cdots \leq f(n)$. If $f(i)<i$ for any $i$, with initial lists for $C(1), C(2), \ldots, C(i)$ fewer than $i$ colors are available for the first $i$ cells and there is no proper coloring. So $f(i) \geq i$ for $i=1,2, \ldots, n$ and the size of $f$ is at least $n(n+1) / 2$. 
For lower bounds on the sum choice number of $2 \times n$ arrays we use a stronger result about $1 \times n$ arrays, showing that if the sum of list sizes is not too large then there exist lists (in fact initial lists will suffice) for which many of the cells in the array have the same color in all proper list colorings. This forced coloring will be used to eliminate colors from other lists in the array.

Let $\mathcal{C}$ be a collection of lists. We will say that a cell is forced by $\mathcal{C}$ if the cell gets the same color in every proper $\mathcal{C}$-coloring.

Lemma 1 Let $f$ be a function with size $t+n(n+1) / 2$ for which the $1 \times n$ array is $f$-choosable. If $\mathcal{C}$ consists of initial lists with sizes specified by $f$ then at least $n-2 t$ cells are forced.

Proof: As discussed above we may assume that $f(i) \geq i$. If $f(i-1)=i-1$ and $f(i)=i$ then using initial lists for $C(1), C(2), \ldots, C(i-1), C(i)$ forces colors $1,2, \ldots, i-1$ to be used in the first $i-1$ cells and color $i$ to be used in the $i^{t h}$ cell. For simplicity in what follows we will assume that there is an $f(0)=0$ so that the condition that the first cell is forced if $f(1)=1$ follows the same pattern as above. If we choose initial lists for all cells then the number of forced cells is at least the number of indices $i$ such that $f(i)=i$ and $f(i-1)=i-1$. Since $\sum_{i=1}^{n}(f(i)-i)=t$ and $(f(i)-i) \geq 0$ for all $i$, there are at most $t$ indices $i$ such that $f(i) \neq i$. Hence there are at most $2 t$ indices $i$ such that either $f(i) \neq i$ or $f(i-1) \neq i-1$. So at most $2 t$ cells are not forced.

Lemma 2 If the $2 \times n$ array is $f$-choosable then the size of $f$ is at least $n^{2}+\lceil 5 n / 3\rceil$.

Proof: From the bounds for the $1 \times n$ array, we may assume that $t_{1}, t_{2} \geq 0$ where $\sum_{i=1}^{n} f(1, i)=t_{1}+n(n+1) / 2$ and $\sum_{i=1}^{n} f(2, i)=t_{2}+n(n+1) / 2$. By switching rows if neccesary we may assume that $t_{1} \leq t_{2}$. With initial lists $\mathcal{C}_{1}$ for the cells in row 1 at least $n-2 t_{1}$ cells of row 1 are forced (using Lemma 1 ). For $i=1,2, \ldots, n$ let $f^{\prime}(i)=f(2, i)$ if cell $(1, i)$ is not forced by $\mathcal{C}_{1}$ and let $f^{\prime}(i)=f(2, i)-1$ if cell $(1, i)$ is forced by $\mathcal{C}_{1}$. Let $\mathcal{C}_{2}^{\prime}$ be a collection of lists for a $1 \times n$ array with sizes specified by $f^{\prime}$. Construct a collection $\mathcal{C}$ of lists for the $2 \times n$ array as follows: By relabeling, we may assume that the colors in the lists $\mathcal{C}_{1}$ are distinct from those in the lists $\mathcal{C}_{2}^{\prime}$. Let the list for the cells in the first row be given by $\mathcal{C}_{1}$. For each forced cell $i$ from the first row call the forced color $c_{i}$. Then let the list for cell $(2, i)$ be the $i^{\text {th }}$ list of $\mathcal{C}_{2}^{\prime}$ if cell $(1, i)$ is not forced let it be the $i^{\text {th }}$ list of $\mathcal{C}_{2}^{\prime}$ along with $c_{i}$ if cell $(1, i)$ is forced. Then the sizes of of the lists in $\mathcal{C}$ are given by $f$ and hence the $2 \times n$ array is $\mathcal{C}$-colorable. This induces a coloring in row 2 in which the forced colors $c_{i}$ are avoided and thus row 2 is $\mathcal{C}_{2}^{\prime}$-colorable. As this holds for any choice of $\mathcal{C}_{2}^{\prime}$ we get that the $1 \times n$ array is $f^{\prime}$-choosable. So the size of $f^{\prime}$ is at least $n(n+1) / 2$ and hence $\sum_{i=1}^{n} f(2, i) \geq\left(n-2 t_{1}\right)+n(n+1) / 2$.

So $t_{2} \geq n-2 t_{1}$ and $t_{1} \leq t_{2}$. Thus $t_{1}+t_{2} \geq t_{1}+\left(n-2 t_{1}\right)=n-t_{1}$ and $t_{1}+t_{2} \geq 2 t_{1}$. Suppose $t_{1}+t_{2}<2 n / 3$. Then $2 n / 3>n-t_{1}$ (so $t_{1}>n / 3$ ) and $2 t_{1}<2 n / 3$ which yields the contradiction $2 n / 3>n-n / 3$. Hence $t_{1}+t_{2} \geq\lceil 2 n / 3\rceil$. Finally we see that the size of $f$ is $n(n+1) / 2+t_{1}+n(n+1) / 2+t_{2} \geq n(n+1)+\lceil 2 n / 3\rceil=n^{2}+\lceil 5 n / 3\rceil$. 


\section{Upper Bound}

To complete the proof of Theorem 1 we need to construct a choosable $f$ with size $n^{2}+$ $\lceil 5 n / 3\rceil$. and show that the $2 \times n$ array is $f$-choosable.

Lemma 3 There exists a choosable $f$ for the $2 \times n$ array with size $n^{2}+\lceil 5 n / 3\rceil$.

Proof: It is straightforward to check that $\begin{aligned} & 1 \\ & 2\end{aligned}$ and $\begin{array}{ll}2 & 3 \\ 1 & 2\end{array}$ are choosable. These show the desired bounds for $n=1,2$.

For $2 \times 3$ arrays, the following assignment of set sizes with sum 14 can be shown to be choosable: $\begin{array}{lll}2 & 2 & 3 \\ 3 & 2 & 2\end{array}$. Checking that this $f$ is choosable can be done with straightforward consideration of cases. The details of this are long and will be given in the next section.

For now we assume this and and consider the following $f$ for a $2 \times n$ array with $n=3 k-1$. For $n=3 k-2$ (or $n=3 k-3$ ) simply delete the last column (or two columns).

$$
\begin{array}{lll|lll|lll|lll|lll|ll}
2 & 2 & 3 & 5 & 5 & 6 & 8 & 8 & 9 \\
3 & 2 & 2 & 6 & 5 & 5 & 9 & 8 & 8 & . & . & \cdot & 3 k-4 & 3 k-4 & 3 k-3 & 3 k-2 & 3 k-1 \\
3 k-3 & 3 k-4 & 3 k-4 & 3 k-1 & 3 k
\end{array} .
$$

Use induction to show that these $f$ are choosable. The base cases are discussed above. If $n>3$ and $\mathcal{C}$ is a collection of lists with sizes specified by $f$ then color the first 3 cells in each row inductively. Exactly 3 colors are used in each row for these cells. Let $T_{i}$ be the set of colors used in row $i$. Then form new sets $C^{\prime}(i, j)=C(i, j+3)-T_{i}$ for $i=1,2$ and $j=1,2 \ldots, n-3$. Since we have $\left|T_{i}\right|=3$ the new sets have sizes that are at least those specified by $f$ for a $2 \times(n-3)$ array. Color these cells using induction and then use these colors to give a proper $\mathcal{C}$-coloring of the original $2 \times n$ array.

\section{The $2 \times 3$ case}

In this section we give the detailed case analysis to show that the $2 \times 3$ array is $\mathcal{C}$-colorable if the list sizes are specified by $f$ given by $\begin{array}{lll}2 & 2 & 3 \\ 3 & 2 & 2\end{array}$. We will attempt partial colorings and refer to reduced lists as those lists obtained by deleting colors that can not be used in completing a proper list coloring.

(i): $C(1,1) \cap C(2,3) \neq \emptyset$. Assume $a$ is in the intersection. Attempt to color with $c_{1,1}=c_{2,3}=a$. Then deleting color $a$ from the remaining lists leaves lists with sizes that

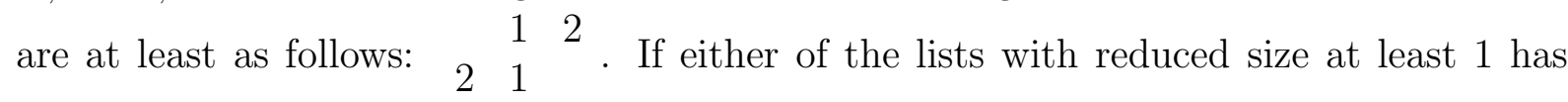
size strictly greater than 1 then we can complete a proper list coloring greedily using any ordering with non-decreasing list sizes. If not, then $a$ is in both of the lists and a list coloring can not be completed only if the reduced lists of size 1 are the same. So we are done unless $C(1,2)=C(2,2)=\{a, x\}$ for some $x$. Let $r$ be the other color in $C(1,1)$, i.e., $C(1,1)=\{a, r\}$ (where possibly $r=x$ ). 
Now we try another coloring without using color $a$ for both $c_{1,1}$ and $c_{2,3}$. Attempt to color $c_{1,1}=r, c_{1,2}=a, c_{2,2}=x$ and $c_{2,3}=a$. The reduced list for $C(1,3)$ still has size at least 1 and thus we can choose $c_{1,3}$ from it. We can complete a proper list coloring by selecting $c_{2,1}$ unless $r \neq x$ and $C(2,1)=\{a, r, x\}$. Now, let $s$ be the other color in $C(2,3)$. Possibly $s=x$ or $s=r$. Attempt to color $c_{2,3}=s$ and $c_{1,1}=a, c_{1,2}=x, c_{2,2}=a$ and set color $c_{2,1}$ to be one of $\{r, x\}$ that is not $s$. Then we can complete the coloring finding $c_{1,3}$ unless $s \neq x$ and $C(1,3)=\{a, x, s\}$. If this is the case, color $c_{1,1}=r, c_{2,1}=c_{1,2}=$ $x, c_{2,2}=c_{1,3}=a, c_{2,3}=s$ and we are done.

(ii): $C(1,1) \cap C(2,2) \neq \emptyset$. (The case $C(2,3) \neq C(1,2)$ is also covered by symmetry.) Assume $a$ is in the intersection. We may also assume that $a$ is not in $C(2,3)$ or else we are done by (i). Attempt to color $c_{1,1}=c_{2,2}=a$. Then deleting color $a$ from every list leaves lists with sizes that are at least as follows: $\begin{array}{rrr}1 & 2 \\ 2 & 2\end{array}$. We can easily complete a proper list coloring by greedily picking colors in the order $c_{1,2}, c_{1,3}, c_{2,3}, c_{2,1}$.

(iii): $C(1,2) \cap C(2,2) \neq \emptyset$. Assume that $a$ is in the intersection. Let $C(1,1)=\{x, y\}$ and $C(2,3)=\{r, s\}$. Applying (i) and (ii) we can assume that $x, y, r, s, a$ are all distinct. Let $b$ be the other color in $C(2,2)$. At least one of $r$ or $s$ is not $b$ so assume $r \neq b$. Attempt to color $c_{1,2}=a, c_{2,2}=b, c_{2,3}=r$. Deleting these colors from the remaining lists (and recalling that $a$ is distinct from $x, y$ ) leaves lists with sizes that are at least as

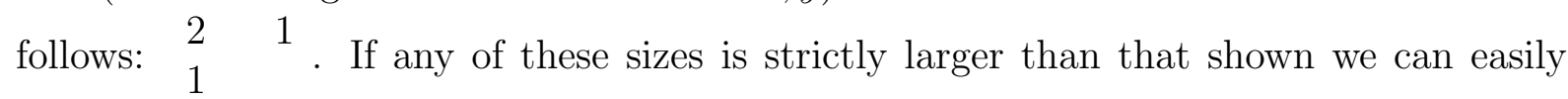
complete a proper list coloring greedily using any ordering with non-decreasing list sizes. So assume $C(1,3) \supset\{a, r\}$ and $C(2,1) \supset\{b, r\}$. We can still complete a proper list coloring unless one of $C(2,1)$ or $C(1,3)$ also contains $x$ and the other $y$. So assume $C(2,1)=\{b, r, y\}$ and $C(1,3)=\{a, r, x\}$. Let $d$ be the other color in $C(1,2)$. At least one of $x$ or $y$ is not $d$ so assume $d \neq x$. Note also that $b \neq x$ and $b \neq y$ by (ii). Then color $c_{1,1}=x, c_{1,2}=d, c_{1,3}=c_{2,2}=a, c_{2,1}=b, c_{2,3}=r$ and we are done.

Now we may assume $C(1,2) \cap C(2,2)=\emptyset$ by (iii). Suppose there exists $a \in C(1,2)$ and $r \in C(2,2)$ such that $a$ is missing from at least one of $C(1,1)$ or $C(1,3)$ and $r$ is missing from at least one of $C(2,1)$ or $C(2,3)$. Then coloring $c_{1,2}=a$ and $c_{2,2}=r$ leaves reduced list sizes at least as large as one of the following: $\begin{array}{llllllll}1 & 3 & & 1 & 3 \\ 3 & 1 & \text { or } & 2 & 2\end{array}$ or $\begin{array}{lll}2 & 2 \\ 3 & 1\end{array}$ or $\begin{array}{ll}2 & 2 \\ 2 & 2\end{array}$. In the first three cases we can complete a proper list coloring greedily using any ordering with non-decreasing list sizes. In the last case we can complete a proper list coloring because the $2 \times 2$ array is 2 -choosable. So, by symmetry we may assume that $C(1,2)=\{a, b\}=C(1,1)$ and $C(1,3)=\{a, b, x\}$. Then color $c_{1,3}=x$. The reduced sizes are then $\begin{array}{lll}2 & 2 \\ 3 & 2 & 1\end{array}$. Greedily color picking colors in the order $c_{2,3}, c_{2,2}, c_{2,1}, c_{1,1}, c_{1,2}$. This coloring will complete a proper list coloring since $C(1,2) \cap C(2,2)=\emptyset$. 


\section{Conclusion}

The sum choice number even for the $3 \times 3$ array is not known. In [5] is is reported that $H$. Taylor has shown that for the $3 \times 3$ array that if all $f(i, j)$ are 3 except $f(1,1)=f(2,2)$ then $f$ is choosable. This gives an upper bound of 25 on the sum choice number. Using this size $25 f$ as a basis and repeating the pattern with appropriate amounts added to each term as is done for the $2 \times 3 f$ in the proof of Lemma 3 yields a choosable $f$ for the $n \times n$ array with size $n^{3}-2 n^{2} / 9$. Using tools like Lemma 1 we can show that the sum choice number for the $3 \times 3$ array must be at least 24 .

Consider now some cases for the generalized Dinitz problem. For the $3 \times 3$ array it is reported (without proof) in [5] that H. Taylor has shown that $f$ is not choosable if all the $f(i, j)$ are 3 except: some $f(i, j)=1$ or two $f(i, j)$ in the same row or column equal to 2 or three $f(i, j)$ in distinct rows and columns equal to 2 . For these $f$ consider the following examples:

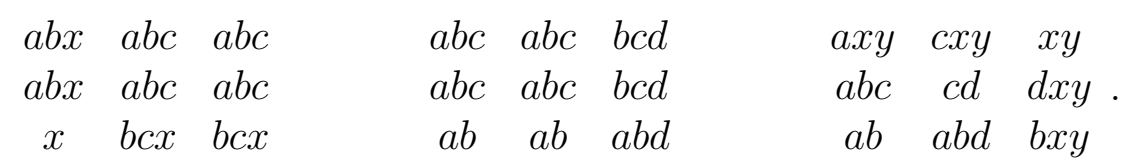

It is straightforward to check that each of these collections of lists does not admit a proper list coloring. The first two examples easily extend to the $n \times n$ case, showing that if the sizes specified by $f$ are all $n$ except for one size 1 or if the sizes specified by $f$ are all $n$ except for $n-1$ sizes $n-1$ all in the same row then $f$ is not choosable.

We do not have a solution to the generalized Dinitz problem (characterize choosable $f$ ) even for $2 \times n$ arrays. The closely related problem of $\mathcal{C}$-coloring, determining for given lists $\mathcal{C}$ if there is a proper $\mathcal{C}$-coloring from those lists is NP-complete for $2 \times n$ arrays ([3] and [4]).

Acknowledgements: The author would like to thank Mike Albertson for useful discussions and the referees for many useful comments.

\section{References}

[1] N. Alon, Restricted colorings of graphs, in Surveys in Combinatorics, K. Walker ed., London Math. Soc. Lecture Notes Series 187, Cambridge Univ. Press, 1992, 1 - 33.

[2] A. Bar-Noy, M.M Halldsrsson, G. Kortsarz, R. Salman and H. Shachnai, Sum multicoloring of graphs. J. Algorithms 37 (2000), 422-450.

[3] D.G. Fon-Der-Flass, Arrays of distinct representatives - a very simple NP-complete problem, Disc. Math. 171 (1997), $295-298$.

[4] A.M. Frieze, Complexity of a 3-dimensional assignment problem, Eur. J. Operations Res. 13 (1983), 161 - 164. 
[5] F. Galvin, The list chromatic index of a bipartite multipartite graph, J. Combin. Theory B 63 (1995), 153-158.

[6] K. Giaro and M. Kubale, Edge-Chromatic sum of trees and bounded cyclicity graphs, Inf. Proc. Letters 75 (2000), 65-69.

[7] A.J.W. Hilton, T. Slivnik and D.S.G. Stirling, Aspects of list coloring, Disc. Math., 231 (2001), $254-264$.

[8] G. Isaak, Sum list edge coloring trees, manuscript, 2001.

[9] J. Kratochvml, Z. Tuza and M. Voigt, New trends in the theory of graph colorings: choosability and list coloring, in Contemporary trends in discrete mathematics, R. Graham et al. eds., DIMACS Ser. Discrete Math. Theoret. Comput. Sci., 49, Amer. Math. Soc., Providence, RI, 1999, 183 - 197.

[10] D.B. West, Introduction to Graph Theory, 2nd ed., Prentice Hall, 2001.

[11] D.R. Woodall, List coloring of graphs, in Surveys in Combinatorics, J.W.P. Hirschfeld ed., London Math. Soc. Lect. Notes Ser. 288, Cambridge Univ. Press, 2001. 